\title{
Síndrome de takotsubo após hemorragia subaracnoidea*
}

Takotsubo cardiomyopathy following subarachnoid hemorrhage

\author{
Eduardo Wajnberg ${ }^{1}$
}

Resumo A síndrome de takotsubo corresponde a uma cardiomiopatia caracterizada por uma disfunção miocárdica transitória afetando ápice do ventrículo esquerdo, que classicamente ocorre após grande estresse físico ou emocional (também chamada "síndrome do coração partido" ou "cardiomiopatia induzida por estresse"). Descrevemos a ocorrência de síndrome de takotsubo em uma paciente induzida por hemorragia subaracnoidea.

Unitermos: Hemorragia subaracnoidea; Aneurisma; Cadiomiopatia de takotsubo.

Abstract Takotsubo cardiomyopathy corresponds to a syndrome characterized by a transient myocardial dysfunction affecting the left ventricular apex that classically occurs after major physical or emotional stress (also called "broken heart syndrome" or "stress-induced cardiomyopathy"). The author describes the case of a patient with takotsubo cardiomyopathy induced by subarachnoid hemorrhage.

Keywords: Subarachnoid hemorrhage; Aneurysm; Takotsubo cardiomyopathy.

Wajnberg E. Síndrome de takotsubo após hemorragia subaracnoidea. Radiol Bras. 2012 Mar/Abr;45(2):132-134.

\section{INTRODUÇÃO}

A síndrome de takotsubo corresponde a uma cardiomiopatia caracterizada por uma disfunção miocárdica transitória afetando ápice do ventrículo esquerdo, que classicamente ocorre após grande estresse físico ou emocional (também chamada de "síndrome do coração partido" ou "cardiomiopatia induzida por estresse") $)^{(\mathbf{1})}$.

Relatamos a ocorrência de síndrome de takotsubo em uma paciente após hemorragia subaracnoidea.

\section{RELATO DO CASO}

Mulher de 55 anos de idade apresentou episódio de cefaleia súbita e intensa, com evidência de hemorragia subaracnoidea grau III de Fisher (Figura 1). Após uma angiografia digital, que demonstrou um aneurisma comunicante anterior de $3 \mathrm{~mm}$ de diâmetro, foi submetida a tratamento endovascular por meio de embolização com

\footnotetext{
* Trabalho realizado no Hospital Universitário Clementino Fraga Filho da Universidade Federal do Rio de Janeiro (HUCFFUFRJ), Rio de Janeiro, RJ, Brasil.

1. Mestre, Médico do Hospital Universitário Clementino Fraga Filho da Universidade Federal do Rio de Janeiro (HUCFF-UFRJ), Rio de Janeiro, RJ, Brasil.

Endereço para correspondência: Dr. Eduardo Wajnberg. Rua Nina Rodrigues, 72/102, Jardim Botânico. Rio de Janeiro, RJ, Brasil, 22461-010. E-mail: eduardowj@gmail.com

Recebido para publicação em 19/7/2011. Aceito, após revisão, em 22/9/2011.
}

micromolas de platina. No período pósoperatório, evoluiu com instabilidade hemodinâmica associada a insuficiência cardíaca esquerda e choque cardiogênico, o qual exigiu o uso de inotrópicos e diuréticos, entubação orotraqueal e ventilação mecânica. O eletrocardiograma (ECG) revelou elevação do segmento ST nas pare- des laterais e prolongamento do intervalo QT. As dosagens de enzimas cardíacas revelaram níveis de troponina e CPK discretamente elevados e o ecocardiograma mostrou redução na fração de ejeção do ventrículo esquerdo associada a acinesia apical e hipercontratilidade basal (Figura 2). A paciente foi submetida a cateterismo car-

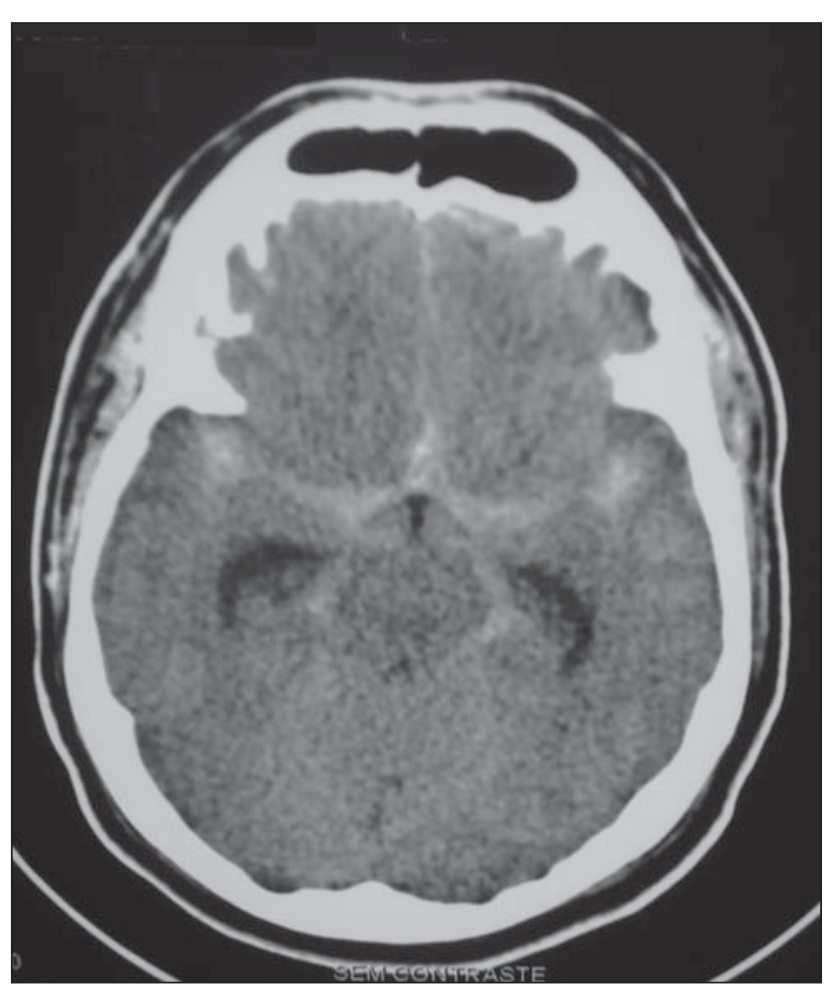

Figura 1. Tomografia computadorizada do crânio revelando hemorragia subaracnoidea difusa, grau III de Fisher, e leve ectasia dos cornos temporais dos ventrículos laterais. 


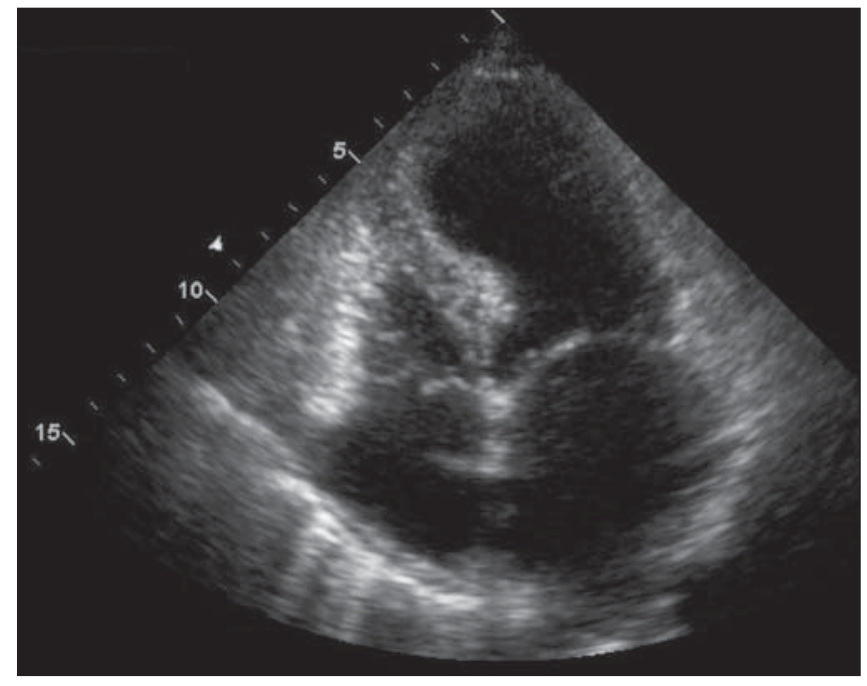

Figura 2. Ecocardiografia em corte coronal quatro câmaras demonstrando o aspecto balonado do ventrículo esquerdo.

díaco, que não revelou lesões ateroscleróticas significativas. A ventriculografia confirmou o aspecto balonado do ventrículo esquerdo, em formato de ânfora, característico da síndrome (Figura 3). No período de cerca de uma semana, a condição clínica foi melhorando e a paciente foi reduzindo as doses de inotrópicos e recuperando a função ventricular esquerda.

\section{DISCUSSÃO}

A síndrome de takotsubo, também chamada de "balonismo apical ventricular esquerdo transitório", foi descrita em 1990 por Sato et al. ${ }^{(2)}$. Seu nome refere-se à aparência do ventrículo esquerdo ao final da sístole na ventriculografia e sua semelhança com a ânfora usada para pegar polvos (do japonês, tako: polvo, tsubo: armadilha Figura 4). É provável que sua etiologia seja multifatorial, mas certamente está envolvida uma falha na autorregulação da microvasculatura miocárdica, vasoespasmo coronariano transitório e uma resposta anormal às catecolaminas liberadas em resposta ao estresse ${ }^{(3)}$. Há forte predominância no sexo feminino, especialmente entre mulheres na pós-menopausa, de $82 \%$ a $100 \%$, com média de idade entre 62 e 75 anos.

Entre os critérios diagnósticos estão incluídos o aspecto balonado do ventrículo

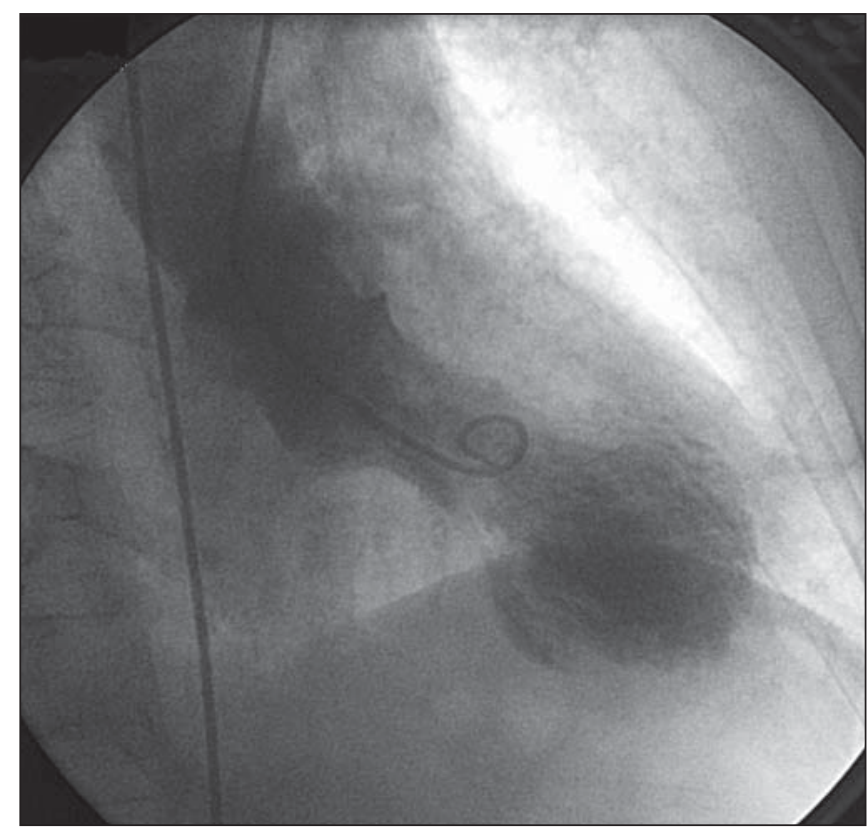

Figura 3. Ventriculografia revelando o aspecto característico da síndrome de takotsubo: o aspecto em ânfora do ventrículo esquerdo ao final da sístole.

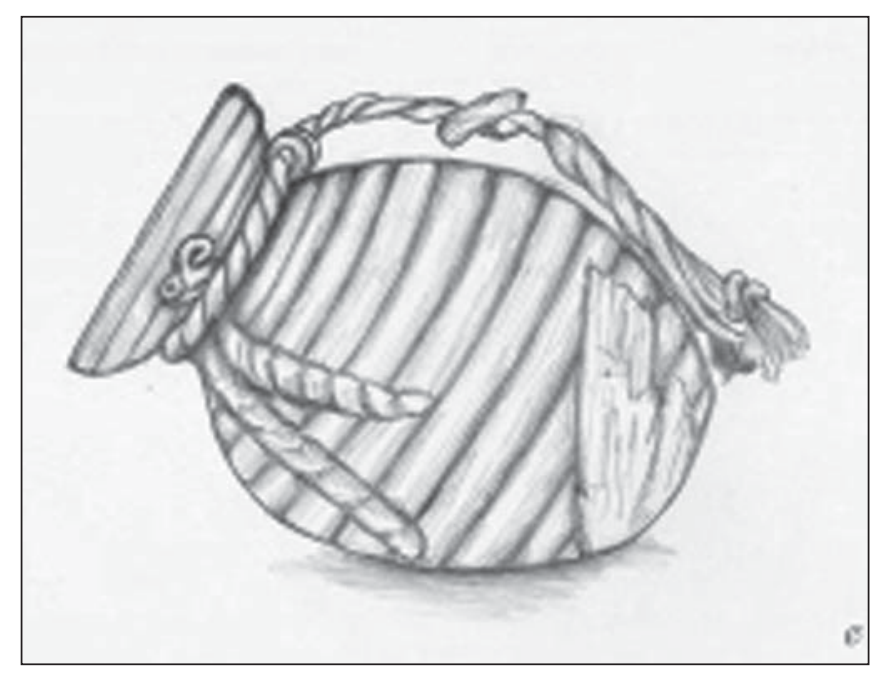

ao ecocardiograma ou ventriculografia, além de hipercontração dos segmentos basais, e alterações do segmento ST e da onda T no ECG. Muitas vezes a síndrome se assemelha ao infarto agudo do miocárdio, em termos de características clínicas, biológicas e de $\mathrm{ECG}^{(4)}$, porém as miocardites e abuso de drogas como a cocaína também entram no diagnóstico diferencial. A ausência de doença arterial coronariana significativa ao cateterismo cardíaco deve sugerir o diagnóstico, e quando suspeito, deve sempre ser realizada a ventriculografia esquerda $^{(5)}$.
A liberação de catecolaminas em excesso desencadeada por estresse físico ou emocional é proposta como o principal mecanismo subjacente na patogênese desse fenômeno, neste caso secundário a hemorragia subaracnoidea. A ressonância magnética cardíaca é promissora no diagnóstico e avaliação desta nova entidade, permitindo a diferenciação entre lesão irreversível, caracterizada pelo realce tardio pelo gadolínio, e edema do miocárdio ${ }^{(6)}$. Dessa forma, a ressonância magnética cardíaca é capaz de acessar a viabilidade miocárdica, sendo importante ferramenta prognóstica ${ }^{(7)}$. De 
modo geral, pode-se dizer que o prognóstico desta enfermidade é bom, com plena recuperação da contratilidade miocárdica depois de alguns dias a semanas ${ }^{(\mathbf{8 , 9})}$.

\section{CONCLUSÃO}

A síndrome de takotsubo é uma doença cada vez mais reconhecida e diagnosticada, e com este relato ressaltamos que também deve ser considerada no diagnóstico diferencial das disfunções miocárdicas que ocorrem no contexto de uma hemorragia intracraniana. Diferentes métodos de imagem desempenham papel primordial no seu diagnóstico, tendo a ressonância magnética um papel promissor em sua avaliação.

\section{REFERÊNCIAS}

1. Gianni M, Dentali F, Grandi AM, et al. Apical ballooning syndrome or takotsubo cardiomyopathy: a systematic review. Eur Heart J. 2006;27:15239.

2. Sato H, Tateishi H, Uchida T, et al. Takotsubo type cardiomyopathy due to multivessel spasm. In: Kodama K, Haze K, Hon M, editors. Clinical aspect of myocardial injury: from ischemia to heart failure. Tokyo: Kagaku Hyoronsha; 1990. p. 5664. [in Japanese].

3. Wittstein IS, Thiemann DR, Lima JA, et al. Neurohumoral features of myocardial stunning due to sudden emotional stress. N Engl J Med. 2005;352: 539-48.

4. Bybee KA, Kara T, Prasad A, et al. Systematic review: transient left ventricular apical ballooning: a syndrome that mimics ST-segment elevation myocardial infarction. Ann Intern Med. 2004;141: 858-65.

5. Azzarelli S, Galassi AR, Amico F, et al. Clinical features of transient left ventricular apical ballooning. Am J Cardiol. 2006;98:1273-6.

6. Fernández-Pérez GC, Aguilar-Arjona JA, de la Fuente GT, et al. Takotsubo cardiomyopathy: assessment with cardiac MRI. AJR Am J Roentgenol. 2010;195:W139-45.

7. Eitel I, Behrendt F, Sareban M, et al. The utility of cardiovascular magnetic resonance imaging in Takotsubo cardiomyopathy (apical ballooning) for differential diagnosis, pathophysiological insights and additional findings. J Cardiovasc Magn Res 2009;11(Suppl 1):O23.

8. Sharkey SW, Windenburg DC, Lesser JR, et al Natural history and expansive clinical profile of stress (tako-tsubo) cardiomyopathy. J Am Coll Cardiol. 2010;55:333-41.

9. Prasad A, Lerman A, Rihal CS. Apical ballooning syndrome (Tako-Tsubo or stress cardiomyopathy) a mimic of acute myocardial infarction. Am Heart J. 2008;155:408-17. 